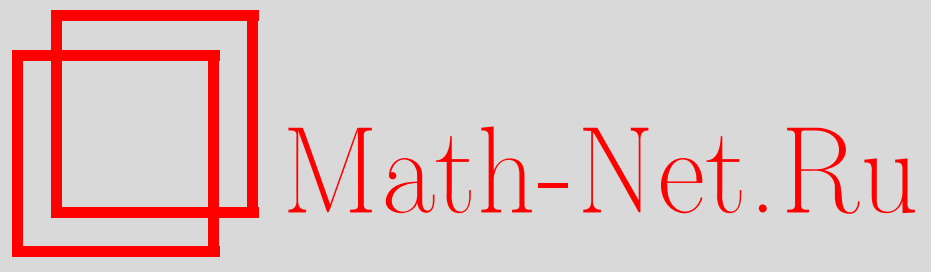

В. Л. Куракин, Инварианты подобия матриц над коммутативным артиновым цепным кольцом, Матем. заметки, 2006, том 80, выпуск 3, 403-412

DOI: https://doi.org/10.4213/mzm2826

Использование Общероссийского математического портала Math-Net.Ru подразумевает, что вы прочитали и согласны с пользовательским соглашением http://www . mathnet.ru/rus/agreement

Параметры загрузки:

IP : 54.162 .85 .209

26 апреля 2023 г., 10:06:29

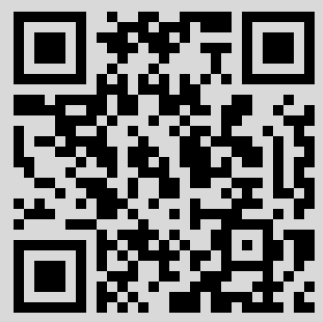




\section{ИНВАРИАНТЫ ПОДОБИЯ МАТРИЦ НАД КОММУТАТИВНЫМ АРТИНОВЫМ ЦЕПНЫМ КОЛЬЦОМ}

\section{В. Л. Куракин}

Квадратной матрице $A$ порядка $m$ над коммутативным артиновым цепным кольцом $R$ ставятся в соответствие $m$ идеалов в кольце многочленов над кольцом дискретного нормирования $S$, гомоморфным образом которого является $R$. Эти идеалы являются инвариантами подобия матриц над кольцом $R$, т.е. они совпадают для подобных матриц. Показывается, что новые инварианты сильнее инвариантов Фиттинга, и что их достаточно для решения задачи о подобии матриц второго порядка над $R$.

Библиография: 4 названия.

Квадратные матрицы $A, B \in R_{m}$ над коммутативным кольцом $R$ с единицей называются подобными, если $C^{-1} A C=B$ для некоторой обратимой матрицы $C \in R_{m}^{*}$. При этом мы пишем $A \approx B$. Фиттинговым инвариантом матрицы $A$ (или матрицы $E x-A)$ называется идеал $D_{k}(E x-A), 1 \leqslant k \leqslant m$, в кольце многочленов $R[x]$, порожденный всеми минорами порядка $k$ характеристической матрицы $E x-A$ (см. [1], [2]). Известно и легко доказывается, что из подобия матриц $A$ и $B$ следует равенство их фиттинговых инвариантов: $D_{k}(E x-A)=D_{k}(E x-B), 1 \leqslant k \leqslant m$ (поэтому говорят, что фиттинговы инварианты являются инвариантами подобия матриц над кольцом $R$ ). Обратное утверждение справедливо для матриц над полем. Если $R-$ поле, то матрица $E x-A$ эквивалентна единственной канонической матрице

$$
E x-A \sim K(E x-A)=\operatorname{diag}\left(f_{1}(x), \ldots, f_{m}(x)\right), \quad f_{i}(x) \mid f_{i+1}(x),
$$

где $f_{i}(x)$ - унитарные многочлены над полем $R$, при этом $D_{k}(E x-A)$ - идеал, порожденный $k$-м инвариантным делителем $f_{1}(x) \cdots f_{k}(x)$ матрицы $E x-A$. Для матриц над коммутативным кольцом $R$, и даже над кольцом вычетов $\mathbb{Z} / p^{n} \mathbb{Z}$, неизвестно простого критерия подобия или аналога канонической формы полиномиальной матрицы.

В этой статье $R$ - коммутативное артиново цепное кольцо, или, эквивалентно, коммутативное артиново локальное кольцо главных идеалов. Единственный максимальный идеал кольца $R$ порождается некоторым элементом $\pi$, и решетка идеалов кольца $R$ есть конечная цепь

$$
R>\pi R>\pi^{2} R>\cdots>\pi^{n-1} R>\pi^{n} R=0,
$$

Работа выполнена при поддержке грантов Президента РФ № МД-2452.2004.10 и № НШ2358.2003.9.

(C) В.Л. Куракин, 2006 
где $n$ - индекс нильпотентности идеала $\pi R$. Обозначим через $\bar{R}=R / \pi R$ поле вычетов кольца $R$.

Согласно [3] кольцо $R$ является гомоморфным образом некоторого кольца дискретного нормирования $S$ с полем вычетов, изоморфным полю $\bar{R}$. Кольцо $S$ является коммутативным нетеровым локальным кольцом главных идеалов, его единственный максимальный идеал порождается некоторым элементом $\omega$, решетка идеалов кольца $S$ есть бесконечная цепь $S>\omega S>\omega^{2} S>\cdots$. Мы будем дополнительно предполагать, что $R \cong S / \omega^{n} S$, т.е. существует эпиморфизм, обладающий следующими свойствами:

$$
\varphi: S \rightarrow R, \quad \varphi(\omega)=\pi, \quad \operatorname{Ker} \varphi=\omega^{n} S .
$$

Например, если $R=\mathbb{Z} / p^{n} \mathbb{Z}$, то в качестве $S$ можно взять кольцо $\mathbb{Z}_{(p)}$ целых $p$ адических чисел, при этом $R \cong S / p^{n} S$. Если кольцо $R$ конечно, то указанное выше условие о том, что $R \cong S / \omega^{n} S$, выполняется [4]. В дальнейшем элемент, порождающий максимальный идеал кольца $S$, будем обозначать той же буквой $\pi$ (вместо $\omega$ ), что и в кольце $R$. Это не будет приводить к двусмысленности, так как всегда будет ясно, в каком кольце, $S$ или $R$, проводятся вычисления. При таком соглашении эпиморфизм $\varphi$ можно назвать эпиморфизмом приведения по модулю $\pi^{n}$.

Эпиморфизм $\varphi$ индуцирует изоморфизм полей вычетов $\bar{\varphi}: \bar{R} \rightarrow \bar{S}$ (который без ограничения общности можно считать тождественным - иначе нужно выбрать другое кольцо $S$ ). Он также индуцирует гомоморфизмы колец многочленов и колец матриц, которые будем обозначать той же буквой $\varphi$ :

$$
\varphi: S[x] \rightarrow R[x], \quad \varphi: S_{m} \rightarrow R_{m} .
$$

Если $A \in R_{m}$, то произвольную матрицу $A_{1} \in S_{m}$ такую, что $\varphi\left(A_{1}\right)=A$, будем называть продолжением матрицы $A$ на кольцо $S$. Для множества всех продолжений матрицы $A$ будем использовать обозначения

$$
[A]=\varphi^{-1}(A)=\left\{A_{1} \in S_{m}: \varphi\left(A_{1}\right)=A\right\}=A_{1}+\pi^{n} S_{m},
$$

где в правой части равенств $A_{1}$ - одно произвольное продолжение матрицы $A$.

Пусть $D_{k}(E x-A)$ - фиттинговы инварианты матрицы $A \in R_{m}$ в кольце $R[x]$, $D_{k}^{S}\left(E x-A_{1}\right)$ - фиттинговы инварианты матрицы $A_{1} \in S_{m}$ в кольце $S[x]$. Последние, конечно, различны для различных продолжений $A_{1} \in[A]$ матрицы $A$. Обозначим через $\widehat{D}_{k}(E x-A)$ идеал кольца $S[x]$, порожденный идеалами $D_{k}^{S}\left(E x-A_{1}\right)$ для всех продолжений $A_{1}$ матрицы $A$ :

$$
\widehat{D}_{k}(E x-A)=\left(D_{k}^{S}\left(E x-A_{1}\right): A_{1} \in[A]\right) .
$$

Это идеал в кольце $S[x]$, однозначно определенный матрицей $A \in R_{m}$. Очевидно,

$$
\varphi\left(\widehat{D}_{k}(E x-A)\right)=\varphi\left(D_{k}^{S}\left(E x-A_{1}\right)\right)=D_{k}(E x-A), \quad A_{1} \in[A],
$$

поэтому идеалы $\widehat{D}_{k}(E x-A)$ являются продолжением (подъемом) фиттинговых инвариантов $D_{k}(E x-A)$ на кольцо $S[x]$.

Следующая теорема показывает, что идеалы (1) являются инвариантами подобия матриц над кольцом $R$. В дальнейшем через $|C|$ обозначается определитель матрицы $C$. 
Теорема 1. Если матрицы $A, B \in R_{m}$ подобнъ, то

$$
\widehat{D}_{k}(E x-A)=\widehat{D}_{k}(E x-B), \quad 1 \leqslant k \leqslant m .
$$

ДокАЗАТЕЛЬСТво. По условию $C^{-1} A C=B$ для некоторой матрицы $C \in R_{m}^{*}$. Пусть $C_{1} \in[C]$ - произвольное продолжение матрицы $C$ на кольцо $S$. Так как $C$ обратима над $R$, элемент $|C|$ обратим в $R$. Тогда элемент $|\bar{C}|$ обратим в поле $\bar{R}$, а значит, $\left|\bar{C}_{1}\right|=|\bar{C}|$ обратим в поле $\bar{S}=\bar{R}$. Следовательно, $C_{1} \in S_{m}^{*}$.

Если $A_{1} \in[A]$, то матрица $B_{1}=C_{1}^{-1} A_{1} C_{1}$ принадлежит $[B]$. Так как матрицы $A_{1}$ и $B_{1}$ подобны над $S$, то их фиттинговы инварианты равны: $D_{k}^{S}\left(E x-A_{1}\right)=$ $D_{k}^{S}\left(E x-B_{1}\right), 1 \leqslant k \leqslant m$. Поэтому из (1) следует, что

$$
\widehat{D}_{k}(E x-A) \subseteq \widehat{D}_{k}(E x-B)
$$

Ввиду обратимости матрицы $C_{1}$ верно и обратное включение.

Отметим, что доказательство теоремы, фактически, основано на том, что отображение $A_{1} \rightarrow C_{1}^{-1} A_{1} C_{1}$ задает взаимно однозначное соответствие между множествами $[A]$ и $[B]$.

В силу теоремы 1 идеалы $\widehat{D}_{k}(E x-A), 1 \leqslant k \leqslant m$, можно называть инвариантами подобия матриц над кольцом $R$. Чтобы отличать их от фиттинговых инвариантов, мы будем называть их "новыми инвариантами". Из равенства (2) видно, что фиттинговы инварианты восстанавливаются по новым инвариантам, поэтому новые инварианты не слабее фиттинговых. В дальнейшем (пример 1) будет показано, что в общем случае новые инварианты сильнее фиттинговых.

Нашей следующей задачей является построение эффективного метода вычисления новых инвариантов.

Лемма 1. Пусть $A \in R_{m}, A_{1} \in[A]$. Тогда

$$
\begin{gathered}
\widehat{D}_{m}(E x-A) \subseteq D_{m}^{S}\left(E x-A_{1}\right)+\pi^{n} D_{m-1}^{S}\left(E x-A_{1}\right)+\pi^{2 n} D_{m-2}^{S}\left(E x-A_{1}\right)+\cdots \\
+\pi^{(m-1) n} D_{1}^{S}\left(E x-A_{1}\right)+\pi^{m n} S[x] .
\end{gathered}
$$

ДокАЗАТЕЛЬСтво. Обозначим правую часть доказываемого включения через $T$. Согласно определению (1) нужно доказать, что если $A_{2} \in[A]$, то $D_{m}^{S}\left(E x-A_{2}\right) \subseteq T$. Так как $D_{m}^{S}\left(E x-A_{2}\right)$ - идеал, порожденный определителем матрицы $E x-A_{2}$, достаточно проверить включение $\left|E x-A_{2}\right| \in T$. Матрицу $A_{2}$ можно представить в виде $A_{2}=A_{1}-\pi^{n} C, C \in S_{m}$. Обозначим $B=E x-A_{1} \in S[x]_{m}$. Тогда $E x-A_{2}=$ $B+\pi^{n} C$, и требуется доказать, что

$$
\begin{gathered}
\left|B+\pi^{n} C\right| \in T=D_{m}^{S}(B)+\pi^{n} D_{m-1}^{S}(B)+\pi^{2 n} D_{m-2}^{S}(B)+\cdots \\
+\pi^{(m-1) n} D_{1}^{S}(B)+\pi^{m n} S[x] .
\end{gathered}
$$

Запишем матрицы $B$ и $C$ по столбцам: $B=\left(B_{1}, \ldots, B_{m}\right), C=\left(C_{1}, \ldots, C_{m}\right)$. Определитель матрицы, один из столбцов которой есть сумма двух столбцов, представляется известным образом как сумма двух определителей. Применяя это свойство, 
получим разложение

$$
\begin{aligned}
\left|B+\pi^{n} C\right| & =\sum_{r=0}^{m} \sum_{1 \leqslant j_{1}<\cdots<j_{r} \leqslant m}\left|B_{1}, \ldots, \pi^{n} C_{j_{1}}, \ldots, \pi^{n} C_{j_{r}}, \ldots, B_{m}\right| \\
& =\sum_{r=0}^{m} \pi^{r n} \sum_{1 \leqslant j_{1}<\cdots<j_{r} \leqslant m}\left|B_{1}, \ldots, C_{j_{1}}, \ldots, C_{j_{r}}, \ldots, B_{m}\right| .
\end{aligned}
$$

Во внутренней сумме разложим каждый определитель по столбцам $j_{1}, \ldots, j_{r}$; при этом минор, стоящий в этих столбцах, совпадет с соответствующим минором матрицы $C$, а его алгебраическое дополнение - с соответствующим алгебраическим дополнением матрицы $B$ :

$$
\left|B+\pi^{n} C\right|=\sum_{r=0}^{m} \pi^{r n} \sum_{1 \leqslant j_{1}<\cdots<j_{r} \leqslant m} \sum_{1 \leqslant i_{1}<\cdots<i_{r} \leqslant m} M_{C}\left(\begin{array}{l}
i_{1} \ldots i_{r} \\
j_{1} \ldots j_{r}
\end{array}\right) \overline{A M}_{B}\left(\begin{array}{c}
i_{1} \ldots i_{r} \\
j_{1} \ldots j_{r}
\end{array}\right) .
$$

Так как

$$
M_{C}\left(\begin{array}{l}
i_{1} \ldots i_{r} \\
j_{1} \ldots j_{r}
\end{array}\right) \in S \subset S[x], \quad \overline{A M}_{B}\left(\begin{array}{l}
i_{1} \ldots i_{r} \\
j_{1} \ldots j_{r}
\end{array}\right) \in D_{m-r}^{S}(B)
$$

то

$$
\left|B+\pi^{n} C\right| \in \sum_{r=0}^{m} \pi^{r n} D_{m-r}^{S}(B)=T,
$$

что и требовалось доказать.

Из доказательства леммы вытекает

СлЕДСТвИЕ. Для любых матрии, $B, C \in S[x]_{m}$ выполняется соотношение (4).

Лемма 2. Пусть $A \in R_{m}, A_{1} \in[A]$. Тогда

$$
\begin{gathered}
\widehat{D}_{m}(E x-A)=D_{m}^{S}\left(E x-A_{1}\right)+\pi^{n} D_{m-1}^{S}\left(E x-A_{1}\right)+\pi^{2 n} D_{m-2}^{S}\left(E x-A_{1}\right)+\cdots \\
+\pi^{(m-1) n} D_{1}^{S}\left(E x-A_{1}\right)+\pi^{m n} S[x] .
\end{gathered}
$$

ДокАЗАтЕльство. Включение $\subseteq$ доказано в лемме 1. Для доказательства обратного включения достаточно проверить, что для $0 \leqslant r \leqslant m$

$$
\pi^{r n} D_{m-r}^{S}\left(E x-A_{1}\right) \subseteq \widehat{D}_{m}(E x-A)=\left(D_{m}^{S}\left(E x-A_{2}\right): A_{2} \in[A]\right),
$$

где $D_{0}^{S}\left(E x-A_{1}\right)$ полагается равным $S[x]$. Обозначим $E x-A_{1}=B$ и будем считать, что $A_{2}=A_{1}-\pi^{n} C, C \in S_{m}$. Тогда доказываемое включение запишется в виде

$$
\pi^{r n} D_{m-r}^{S}(B) \subseteq\left(\left|B+\pi^{n} C\right|: C \in S_{m}\right), \quad 0 \leqslant r \leqslant m
$$

Обозначим правую часть через $Q$ и докажем включение индукцией по $r$.

Взяв $C=0$, получим, что $|B| \in Q$, что доказывает базис индукции при $r=0$.

Пусть $C=E_{i j}$ - матрица, у которой на пересечении $i$-й строки и $j$-го столбца находится единица, а остальные элементы равны нулю. Тогда

$$
\left|B+\pi^{n} C\right|=\left|B+\pi^{n} E_{i j}\right|=|B|+\pi^{n} \overline{A M}_{B}\left(\begin{array}{l}
i \\
j
\end{array}\right) \in Q
$$


Так как $|B| \in Q$ и $Q$ - идеал, то $\pi^{n} \overline{A M}_{B}\left(\begin{array}{l}i \\ j\end{array}\right) \in Q$ для любых $i, j$. Следовательно, любой минор порядка $m-1$ матрицы $B$, умноженный на $\pi^{n}$, принадлежит $Q$. Это означает, что $\pi^{n} D_{m-1}(B) \subseteq Q$.

Возьмем теперь в качестве $C$ матрицу, у которой подматрица размеров $2 \times 2$, стоящая на пересечении строк $i_{1}, i_{2}$ и столбцов $j_{1}, j_{2}$, есть единичная матрица, а остальные элементы равны нулю. Тогда

$$
\begin{aligned}
\mid B+ & \pi^{n} C|=| \ldots, B_{j_{1}}+\pi^{n} C_{j_{1}}, \ldots, B_{j_{2}}+\pi^{n} C_{j_{2}}, \ldots \mid \\
& =|B|+\pi^{n}\left|\ldots C_{j_{1}} \ldots B_{j_{2}} \ldots\right|+\pi^{n}\left|\ldots B_{j_{1}} \ldots C_{j_{2}} \ldots\right|+\pi^{2 n}\left|\ldots C_{j_{1}} \ldots C_{j_{2}} \ldots\right| \\
& =|B|+\pi^{n} \overline{A M}_{B}\left(\begin{array}{c}
i_{1} \\
j_{1}
\end{array}\right)+\pi^{n} \overline{A M}_{B}\left(\begin{array}{c}
i_{2} \\
j_{2}
\end{array}\right)+\pi^{2 n} \overline{A M}_{B}\left(\begin{array}{ll}
i_{1} & i_{2} \\
j_{1} & j_{2}
\end{array}\right) \in Q,
\end{aligned}
$$

где неуказанные столбцы являются столбцами матрцы $B$. С учетом уже доказанного $\pi^{2 n} \overline{A M}_{B}\left(\begin{array}{ll}i_{1} & i_{2} \\ j_{1} & j_{2}\end{array}\right) \in Q$. Поэтому все миноры порядка $m-2$ матрицы $B$, умноженные на $\pi^{2 n}$, принадлежат $Q$. Следовательно, $\pi^{2 n} D_{m-2}(B) \subseteq Q$.

Рассуждая аналогично, докажем включение (5) для $r=3, \ldots, m-1$. Наконец, полагая $C=E-$ единичной матрице, аналогично получим

$$
\left|B+\pi^{n} C\right|=|B|+\cdots+\pi^{m n}|C|=|B|+\cdots+\pi^{m n} \in Q,
$$

где слагаемые, обозначенные как . ., принадлежат $Q$ по уже доказанному. Отсюда $\pi^{m n} \in Q$, и мы получили включение (5) для $r=m$.

Из доказательства леммы вытекает

СлеДСтвиЕ. Для любой матрищъ $B \in S[x]_{m}$ выполняется соотношение (5).

Теорема 2. Пусть $A \in S_{m} u 1 \leqslant k \leqslant m$. Тогда для любой матрицы $A_{1} \in[A]$

$$
\begin{gathered}
\widehat{D}_{k}(E x-A)=D_{k}^{S}\left(E x-A_{1}\right)+\pi^{n} D_{k-1}^{S}\left(E x-A_{1}\right)+\pi^{2 n} D_{k-2}^{S}\left(E x-A_{1}\right)+\cdots \\
+\pi^{(k-1) n} D_{1}^{S}\left(E x-A_{1}\right)+\pi^{k n} S[x] .
\end{gathered}
$$

ДокАзАтельство. Для $k=m$ теорема доказана в лемме 2. Докажем, что теорема верна для остальных $k$. Обозначим $E x-A_{1}=B \in S[x]_{m}$. Тогда

$$
\widehat{D}_{k}(E x-A)=\left(D_{k}^{S}\left(B+\pi^{n} C\right): C \in S_{m}\right) .
$$

Правую часть доказываемого равенства обозначим $T_{k}$.

Чтобы доказать включение $\subseteq$, достаточно проверить, что для любой матрицы $C \in S_{m}$ любой минор порядка $k$ матрицы $B+\pi^{n} C$ принадлежит $T_{k}$. Пусть $B^{\prime}, C^{\prime} \in$ $S[x]_{k}$ - соответствующие подматрицы матриц $B$ и $C$, так что минор, о котором идет речь, равен $\left|B^{\prime}+\pi^{n} C^{\prime}\right|$. В силу следствия леммы 1 , примененному к матрицам $B^{\prime}$ и $C^{\prime}$,

$$
\left|B^{\prime}+\pi^{n} C^{\prime}\right| \in \sum_{r=0}^{k} \pi^{r n} D_{k-r}^{S}\left(B^{\prime}\right) .
$$

Так как, очевидно, $D_{k-r}^{S}\left(B^{\prime}\right) \subseteq D_{k-r}^{S}(B)$, то

$$
\left|B^{\prime}+\pi^{n} C^{\prime}\right| \in \sum_{r=0}^{k} \pi^{r n} D_{k-r}^{S}(B)=T_{k},
$$


что и требовалось доказать.

Для доказательства обратного включения достаточно проверить, что

$$
\pi^{r n} D_{k-r}^{S}(B) \subseteq \widehat{D}_{k}(E x-A)=\left(D_{k}^{S}\left(B+\pi^{n} C\right): C \in S_{m}\right)
$$

для $0 \leqslant r \leqslant k$. Действительно, пусть $B^{\prime}-$ произвольная $k \times k$ подматрица матрицы $B$. В силу следствия леммы 2 , примененного к матрице $B^{\prime}$,

$$
\pi^{r n} D_{k-r}^{S}\left(B^{\prime}\right) \subseteq\left(\left|B^{\prime}+\pi^{n} C^{\prime}\right|: C^{\prime} \in S_{k}\right)
$$

для $0 \leqslant r \leqslant k$. Отсюда

$$
\pi^{r n} D_{k-r}^{S}\left(B^{\prime}\right) \subseteq\left(D_{k}^{S}\left(B+\pi^{n} C\right): C \in S_{m}\right) .
$$

Так как это включение выполняется для любой $k \times k$ подматрицы $B^{\prime}$ матрицы $B$, то

$$
\pi^{r n} D_{k-r}^{S}(B) \subseteq\left(D_{k}^{S}\left(B+\pi^{n} C\right): C \in S_{m}\right)
$$

что и требовалось доказать.

Приведем примеры вычисления новых инвариантов $\widehat{D}_{k}(E x-A)$ с помощью теоремы 2. Следующий пример показывает, что новые инварианты сильнее фиттинговых.

ПримеР 1. Приведем пример матриц $A$ и $B$ таких, что их фиттинговы инварианты совпадают, а новые инварианты различны. Рассмотрим матрицы $2 \times 2$ над кольцом $R=\mathbb{Z} / p^{2} \mathbb{Z}$ :

$$
A=\left(\begin{array}{cc}
0 & p \\
0 & 0
\end{array}\right), \quad B=\left(\begin{array}{ll}
0 & p \\
p & 0
\end{array}\right)
$$

Тогда

$$
E x-A=\left(\begin{array}{cc}
x & -p \\
0 & x
\end{array}\right), \quad E x-B=\left(\begin{array}{cc}
x & -p \\
-p & x
\end{array}\right) .
$$

Фиттинговы инварианты матриц $A$ и $B$ над кольцом $R$ совпадают:

$$
\begin{array}{ll}
D_{1}(E x-A)=(x, p), & D_{2}(E x-A)=\left(x^{2}\right), \\
D_{1}(E x-B)=(x, p), & D_{2}(E x-B)=\left(x^{2}\right) .
\end{array}
$$

Над кольцом $S=\mathbb{Z}_{(p)}$ целых $p$-адических чисел рассмотрим продолжения $A_{1}$ и $B_{1}$ матриц $A$ и $B$ :

$$
A_{1}=\left(\begin{array}{ll}
0 & p \\
0 & 0
\end{array}\right), \quad B_{1}=\left(\begin{array}{cc}
0 & p \\
p & 0
\end{array}\right) .
$$

Внешне эти матрицы совпадают с матрицами $A$ и $B$, но теперь элементы этих матриц принадлежат кольцу $S$, так что $p^{2} \neq 0$. Фиттинговы инварианты матриц $A_{1}$ и $B_{1}$ над кольцом $S$ следующие:

$$
\begin{array}{ll}
D_{1}^{S}\left(E x-A_{1}\right)=(x, p), & D_{2}^{S}\left(E x-A_{1}\right)=\left(x^{2}\right), \\
D_{1}^{S}\left(E x-B_{1}\right)=(x, p), & D_{2}^{S}\left(E x-B_{1}\right)=\left(x^{2}-p^{2}\right) .
\end{array}
$$


Заметим, что эти идеалы являются инвариантами подобия матриц $A_{1}$ и $B_{1}$ над кольцом $S$, но не матриц $A$ и $B$ над кольцом $R$. Теперь с помощью теоремы 2 находим новые инварианты матриц $A$ и $B$ :

$$
\begin{aligned}
& \widehat{D}_{1}(E x-A)=(x, p)+\left(p^{2}\right)=(x, p), \\
& \widehat{D}_{2}(E x-A)=\left(x^{2}\right)+p^{2}(x, p)+\left(p^{4}\right)=\left(x^{2}, p^{2} x, p^{3}\right), \\
& \widehat{D}_{1}(E x-B)=(x, p)+\left(p^{2}\right)=(x, p), \\
& \widehat{D}_{2}(E x-B)=\left(x^{2}+p^{2}\right)+p^{2}(x, p)+\left(p^{4}\right)=\left(x^{2}+p^{2}, p^{2} x, p^{3}\right) .
\end{aligned}
$$

Таким образом, новые инварианты матриц $A$ и $B$ различны, в то время как фиттинговы инварианты совпадают. По теореме 1 матрицы $A$ и $B$ над кольцом $R$ не подобны. Данный пример говорит о том, что новые инварианты сильнее фиттинговых инвариантов.

Из примера можно увидеть, за счет чего новые инварианты оказываются сильнее фиттинговых. Сравнивая соотношения (6) и (7), мы получаем, что в кольце $R$ минор $|E x-B|$ равен $x^{2}-p^{2}=x^{2}$, т.е. слагаемое $p^{2}$ исчезает. При переходе к кольцу $S$ это слагаемое остается, так что $\left|E x-B_{1}\right|=x^{2}-p^{2} \neq x^{2}$.

В приведенном примере матрицы $A$ и $B$ вырожденные, и даже нулевые по модулю $p$. Аналогичный пример можно привести и для невырожденных, например, рассмотрев матрицы

$$
E+A=\left(\begin{array}{ll}
1 & p \\
0 & 1
\end{array}\right), \quad E+B=\left(\begin{array}{ll}
1 & p \\
p & 1
\end{array}\right) .
$$

Как показывает следующий пример, новые инварианты не образуют полную систему инвариантов подобия матриц над кольцом $R$.

ПримеР 2. Существуют матрицы, не являющиеся подобными, новые инварианты которых совпадают. Возьмем кольцо $R=\mathbb{Z} / p^{2} \mathbb{Z}$ и матрицы

$$
A=\left(\begin{array}{ccc}
0 & p & 0 \\
0 & 0 & 1 \\
0 & 0 & p
\end{array}\right), \quad B=\left(\begin{array}{ccc}
0 & 0 & 0 \\
0 & 0 & 1 \\
p & 0 & p
\end{array}\right)
$$

Над кольцом $S=\mathbb{Z}_{(p)}$ рассмотрим продолжения $A_{1}$ и $B_{1}$ матриц $A$ и $B:$

$$
A_{1}=\left(\begin{array}{ccc}
0 & p & 0 \\
0 & 0 & 1 \\
0 & 0 & p
\end{array}\right), \quad B_{1}=\left(\begin{array}{ccc}
0 & 0 & 0 \\
0 & 0 & 1 \\
p & 0 & p
\end{array}\right)
$$

Тогда

$$
E x-A_{1}=\left(\begin{array}{ccc}
x & -p & 0 \\
0 & x & -1 \\
0 & 0 & x-p
\end{array}\right), \quad E x-B_{1}=\left(\begin{array}{ccc}
x & 0 & 0 \\
0 & x & -1 \\
-p & 0 & x-p
\end{array}\right) .
$$

Фиттинговы инварианты этих матриц над кольцом $S$ таковы:

$$
\begin{array}{ll}
D_{1}^{S}\left(E x-A_{1}\right)=(1), & D_{2}^{S}\left(E x-A_{1}\right)=(x, p), \quad D_{3}^{S}\left(E x-A_{1}\right)=\left(\chi_{A_{1}}(x)\right)=\left(x^{3}-p x^{2}\right), \\
D_{1}^{S}\left(E x-B_{1}\right)=(1), & D_{2}^{S}\left(E x-B_{1}\right)=(x, p), \quad D_{3}^{S}\left(E x-B_{1}\right)=\left(\chi_{B_{1}}(x)\right)=\left(x^{3}-p x^{2}\right) .
\end{array}
$$


Таким образом, $D_{k}^{S}\left(E x-A_{1}\right)=D_{k}^{S}\left(E x-B_{1}\right), k=1,2,3$. В силу теоремы 2 отсюда следует, что

$$
\widehat{D}_{k}^{S}\left(E x-A_{1}\right)=\widehat{D}_{k}^{S}\left(E x-B_{1}\right), \quad k=1,2,3,
$$

т.е. новые инварианты матриц $A$ и $B$ совпадают. С другой стороны, матрицы $A$ и $B$ над кольцом $R$ не подобны. Действительно, предположим, что $C^{-1} A C=B$ для некоторой матрицы $C \in R_{3}^{*}$. Обозначим

$$
C=\left(\begin{array}{lll}
a & b & c \\
d & e & f \\
g & h & i
\end{array}\right)
$$

Тогда равенство $A C=C B$ запишется в виде

$$
\left(\begin{array}{ccc}
p d & p e & p f \\
g & h & i \\
p g & p h & p i
\end{array}\right)=\left(\begin{array}{ccc}
p c & 0 & b+p c \\
p f & 0 & e+p f \\
p i & 0 & h+p i
\end{array}\right) .
$$

Отсюда видно, что $p \mid b, p e=0, h=0$. Следовательно, $\bar{b}=\bar{e}=\bar{h}=\overline{0}$, и матрица $C$ не обратима; противоречие.

Как показывает следующая теорема, аналогичный пример нельзя построить для матриц размеров $2 \times 2$. Если $G(x)=x^{m}-c_{m-1} x^{m-1}-\cdots-c_{1} x-c_{0} \in R[x]-$ унитарный многочлен, то обозначим через $S(G)$ сопровождающую матрицу многочлена $G(x)$ :

$$
S(G)=\left(\begin{array}{cccccc}
0 & 0 & \ldots & 0 & 0 & c_{0} \\
1 & 0 & \ldots & 0 & 0 & c_{1} \\
0 & 1 & \ldots & 0 & 0 & c_{2} \\
\ldots \ldots \ldots & \ldots \ldots \ldots \ldots \ldots \ldots \\
0 & 0 & \ldots & 1 & 0 & c_{m-2} \\
0 & 0 & \ldots & 0 & 1 & c_{m-1}
\end{array}\right) .
$$

ТЕОРема 3. Новых инвариантов достаточно для решения задачи подобия матриц, второго порядка над колъцом $R$. Другими словами, матрицы $A, B \in R_{2}$ подобнъь тогда и толъко тогда, когда

$$
\widehat{D}_{k}(E x-A)=\widehat{D}_{k}(E x-B), \quad k=1,2 .
$$

ДоКАЗАТЕЛЬСтво. Проблема подобия матриц над кольцом $R$ сводится к проблеме подобия матриц $A$ со свойством $\bar{A} \neq \overline{0}$, поскольку если $A=\pi A^{\prime}, B=\pi B^{\prime}$, то $A \approx B$ над $R$ тогда и только тогда, когда $A^{\prime} \approx B^{\prime}$ над кольцом $R / \pi^{n-1} R$ (см. [2; с. 97]). Поэтому можно считать, что $A, B \in R_{2}, \bar{A} \neq \overline{0}, \bar{B} \neq \overline{0}$. Так как необходимость следует из теоремы 1 , нужно доказать лишь достаточность. Итак, пусть выполняется условие (8). Требуется доказать, что $A \approx B$.

Согласно [2; теорема 10] матрица $A$ подобна одной из следующих матриц:

I) $A \approx S\left(\chi_{A}(x)\right)$

II) $A=r E, r \in R$;

III) $A \approx r E+\pi^{k} S(G), 0<k<n, G(x)=x^{2}-a x-b$.

Фиттинговы инварианты этих матриц следующие:

I) $D_{1}(E x-A)=(1), D_{2}(E x-A)=\left(\chi_{A}(x)\right)$; 
II) $D_{1}(E x-A)=(x-r), D_{2}(E x-A)=\left((x-r)^{2}\right)$;

III) $D_{1}(E x-A)=\left(x-r, \pi^{k}\right), D_{2}(E x-A)=\left((x-r)^{2}-\pi^{k} a(x-r)-\pi^{2 k} b\right)$.

Первый фиттингов инвариант однозначно определяет группу I, II или III, к которой принадлежит матрица $A$. Внутри групп I и II второй фиттингов инвариант однозначно определяет матрицу $A$ с точностью до подобия. В этих случаях из равенства фиттинговых инвариантов (и тем более из равенства новых инвариантов) следует подобие матриц.

Пусть теперь матрицы $A$ и $B$ принадлежат группе III. Можно считать, что

$$
A=r E+\pi^{k} S\left(x^{2}-a x-b\right), \quad B=r^{\prime} E+\pi^{k^{\prime}} S\left(x^{2}-a^{\prime} x-b^{\prime}\right) .
$$

Так как выполняются условия $(8)$, то $D_{1}(E x-A)=D_{1}(E x-B)$, т.е. $\left(x-r, \pi^{k}\right)=$ $\left(x-r^{\prime}, \pi^{k^{\prime}}\right)$. Отсюда

$$
k^{\prime}=k, \quad r^{\prime}=r+\pi^{k} s, \quad s \in R .
$$

Следовательно,

$$
A=r E+\pi^{k} S\left(x^{2}-a x-b\right), \quad B=\left(r+\pi^{k} s\right) E+\pi^{k} S\left(x^{2}-a^{\prime} x-b^{\prime}\right) .
$$

Над кольцом дискретного нормирования $S$ рассмотрим продолжения $A_{1}$ и $B_{1}$ матриц $A$ и $B$ вида

$$
A_{1}=r E+\pi^{k} S\left(x^{2}-a x-b\right), \quad B_{1}=\left(r+\pi^{k} s\right) E+\pi^{k} S\left(x^{2}-a^{\prime} x-b^{\prime}\right) .
$$

Тогда

$$
\begin{aligned}
& E x-A_{1}=\left(\begin{array}{cc}
x-r & -\pi^{k} b \\
-\pi^{k} & x-r-\pi^{k} a
\end{array}\right) \\
& E x-B_{1}=\left(\begin{array}{cc}
x-r-\pi^{k} s & -\pi^{k} b^{\prime} \\
-\pi^{k} & x-r-\pi^{k} s-\pi^{k} a^{\prime}
\end{array}\right) .
\end{aligned}
$$

Фиттинговы инварианты этих матриц над кольцом $S$ следующие:

$$
\begin{aligned}
& D_{1}^{S}\left(E x-A_{1}\right)=\left(x-r, \pi^{k}\right), \\
& D_{2}^{S}\left(E x-A_{1}\right)=\left(\chi_{A_{1}}(x)\right)=\left((x-r)^{2}-\pi^{k} a(x-r)-\pi^{2 k} b\right), \\
& D_{1}^{S}\left(E x-B_{1}\right)=\left(x-r, \pi^{k}\right), \\
& D_{2}^{S}\left(E x-B_{1}\right)=\left(\chi_{B_{1}}(x)\right)=\left((x-r)^{2}-\pi^{k}\left(a^{\prime}+2 s\right)(x-r)-\pi^{2 k}\left(b^{\prime}-s\left(a^{\prime}+s\right)\right)\right) .
\end{aligned}
$$

$\mathrm{C}$ помощью теоремы 2 находим новые инварианты матриц $A$ и $B$ над кольцом $S$ :

$$
\begin{aligned}
& \widehat{D}_{1}(E x-A)=\left(x-r, \pi^{k}\right), \\
& \widehat{D}_{2}(E x-A)=\left((x-r)^{2}-\pi^{k} a(x-r)-\pi^{2 k} b, \pi^{n}(x-r), \pi^{n+k}\right), \\
& \widehat{D}_{1}(E x-B)=\left(x-r, \pi^{k}\right), \\
& \widehat{D}_{2}(E x-B)=\left((x-r)^{2}-\pi^{k}\left(a^{\prime}+2 s\right)(x-r)-\pi^{2 k}\left(b^{\prime}-s\left(a^{\prime}+s\right)\right), \pi^{n}(x-r), \pi^{n+k}\right) .
\end{aligned}
$$

Из равенства $\widehat{D}_{2}(E x-A)=\widehat{D}_{2}(E x-B)$ получаем, что многочлен $F(x)=\chi_{A_{1}}(x)-$ $\chi_{B_{1}}(x)$ принадлежит $\widehat{D}_{2}(E x-A)$. Так как степень многочлена $F(x)$ равна 1 , то $F(x) \in\left(\pi^{n}(x-r), \pi^{n+k}\right)$ или

$$
F(x)=\pi^{k}\left(a^{\prime}-a+2 s\right)(x-r)+\pi^{2 k}\left(b^{\prime}-b-s\left(a^{\prime}+s\right)\right) \in\left(\pi^{n}(x-r), \pi^{n+k}\right) .
$$


Это равносильно тому, что

$$
a^{\prime}-a+2 s \in\left(\pi^{n-k}\right), \quad b^{\prime}-b-s\left(a^{\prime}+s\right) \in\left(\pi^{n-k}\right),
$$

т.e.

$$
a^{\prime}=a-2 s+\pi^{n-k} \alpha, \quad b^{\prime}=b+s(a-s)+\pi^{n-k} \beta,
$$

где $\alpha, \beta \in S$. Вернемся теперь к матрицам $A$ и $B$ над кольцом $R$ из соотношения (9). В силу доказанного, эти матрицы имеют вид

$$
A=\left(\begin{array}{cc}
r & \pi^{k} b \\
\pi^{k} & r+\pi^{k} a
\end{array}\right), \quad B=\left(\begin{array}{cc}
r+\pi^{k} s & \pi^{k} b+\pi^{k} s(a-s) \\
\pi^{k} & r+\pi^{k} a-\pi^{k} s
\end{array}\right) .
$$

Непосредственно проверяется, что $C^{-1} A C=B$, где

$$
C^{-1}=\left(\begin{array}{ll}
1 & s \\
0 & 1
\end{array}\right), \quad C=\left(\begin{array}{cc}
1 & -s \\
0 & 1
\end{array}\right) .
$$

Следовательно, матрицы $A$ и $B$ над кольцом $R$ подобны.

Таким образом, новые инварианты сильнее инвариантов Фиттинга. Их достаточно для решения задачи о подобии матриц порядка 2, но недостаточно - для матриц порядка 3.

Автор признателен рецензенту за полезные замечания по тексту статьи.

\section{СПИСОК ЦИТИРОВАННОЙ ЛИТЕРАТУРЫ}

[1] H. Fitting, "Die Determinantenideale eines Modulus", Jahresber. Deutch. Math.-Verein., 46 (1936), 195-228.

[2] А. А. Нечаев, "О подобии матриц над коммутативным локальным артиновым кольцом", Тр. семинара им. И. Г. Петровского, 9 (1983), 81-101.

[3] I. S. Cohen, "On the structure and ideal theory of complete local rings", Trans. Amer. Math. Soc., 59:1 (1946), 54-106.

[4] А. А. Нечаев, "О строении конечных коммутативных колец с единицей", Матем. заметки, 10:6 (1971), 679-688.

\section{В. Л. Куракин}

Институт криптографии, связи и информации

Академии ФСБ России

E-mail: kurakin@do.tochka.ru
Поступило

13.05 .2005

Исправленный вариант

24.01.2006 